\title{
Grain-size-dependent thermal conductivity of nanocrystalline yttria- stabilized zirconia films grown by metal-organic chemical vapor deposition
}

\author{
G. Soyez, ${ }^{\text {a) }}$ J. A. Eastman, ${ }^{\text {b) }}$ L. J. Thompson, G.-R. Bai, P. M. Baldo, \\ and A. W. McCormick \\ Materials Science Division, Argonne National Laboratory, Argonne, Illinois 60439 \\ R. J. DiMelfi \\ Reactor Engineering Division, Argonne National Laboratory, Argonne, Illinois 60439 \\ A. A. Elmustafa, M. F. Tambwe, and D. S. Stone \\ Department of Materials Science and Engineering, University of Wisconsin, Madison, Wisconsin 53706
}

(Received 24 May 2000; accepted for publication 5 July 2000)

\begin{abstract}
A grain-size-dependent reduction in the room-temperature thermal conductivity of nanocrystalline yttria-stabilized zirconia is reported for the first time. Films were grown by metal-organic chemical vapor deposition with controlled grain sizes from 10 to $100 \mathrm{~nm}$. For grain sizes smaller than approximately $30 \mathrm{~nm}$, a substantial reduction in thermal conductivity was observed, reaching a value of less than one-third the bulk value at the smallest grain sizes measured. The observed behavior is consistent with expectations based on an estimation of the phonon mean-free path in zirconia. () 2000 American Institute of Physics. [S0003-6951(00)05034-8]
\end{abstract}

The efficiency of gas turbine engines is dictated by the maximum sustained operating temperature of their typically Ni- or Co-based alloy turbine rotors. The development of new, higher temperature, high-strength, lightweight alloys is desirable. ${ }^{1}$ However, recent studies have concluded that significant near-term progress in increasing turbine engine operating temperatures is more likely to come from the development of improved thermal barrier coatings (TBCs), typically yttria-stabilized zirconia (YSZ), than from the design of new alloys. ${ }^{2}$ New processing techniques that result in TBC microstructures with lower thermal conductivity could lead either to higher operating temperatures of turbine engines, resulting in greater efficiency, or thinner coatings for the same operating temperature, which would reduce overall weight. Nanocrystalline YSZ coatings are of interest because they offer the possibility of lowering thermal conductivity, and may also provide additional benefits for TBC applications because of the possibility of improved toughness and ductility compared to that of coarser-grained ceramics. ${ }^{3,4}$

The low thermal conductivity of YSZ $(\sim 2.3 \mathrm{~W} / \mathrm{mK}$ for high-density, polycrystalline material with a yttria-content of $10 \mathrm{~mol} . \%$ at $20^{\circ} \mathrm{C}^{5}$ ) is due primarily to phonon scattering by vacancies on the material's highly defective oxygen sublattice. ${ }^{6}$ The potential for reduced thermal conductivity in nanocrystalline coatings arises from the predicted enhanced phonon scattering due to the presence of numerous closely spaced grain boundaries. For example, Klemens and Gell ${ }^{6}$ have theoretically predicted that the room temperature thermal conductivity of $10 \mathrm{~nm}$ grain-sized YSZ containing 7 wt. $\% \mathrm{Y}_{2} \mathrm{O}_{3}$ will be decreased more than $50 \%$ compared to 1 $\mu \mathrm{m}$ grain-sized YSZ of the same composition. The goal of the present study was to experimentally determine the effect

${ }^{a)}$ Present affiliation: Carl Zeiss, Oberkochen, Germany.

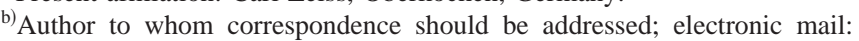
jeastman@anl.gov of grain size on the room-temperature thermal conductivity of YSZ, thus contributing to the fundamental understanding of grain-size-dependent phonon scattering processes.

Nanocrystalline YSZ films were grown by metal-organic chemical vapor deposition (MOCVD) using a low-pressure, horizontal, cold-walled deposition system. Yttrium $b$-diketonate $\left[\mathrm{Y}(\text { thd })_{3}\right]$ and zirconium $t$-butoxide $\left[\mathrm{ZrOC}\left(\mathrm{CH}_{3}\right)_{4}\right]^{7}$ were chosen as precursor materials. Highpurity nitrogen was used as the precursor carrier gas. The precursors were mixed with high-purity oxygen and nitrogen in the quartz deposition chamber, which was maintained at 5 Torr during all growth experiments. Samples were grown on polished polycrystalline $\alpha-\mathrm{Al}_{2} \mathrm{O}_{3}$ substrates at temperatures of $500-600{ }^{\circ} \mathrm{C}$. Typical growth rates were $\sim 0.5 \mu \mathrm{m} / \mathrm{h}$, with resulting film thicknesses between 0.5 and $1.2 \mu \mathrm{m}$ (measured by Rutherford backscattering spectroscopy). Following YSZ film growth, a $\sim 300$-nm-thick, $25-\mu \mathrm{m}$-wide $\mathrm{Au} / \mathrm{Cr}$ heater line was formed on each sample by electron beam evaporation followed by photolithography and wet chemical etching techniques.

Samples were characterized by $\mathrm{x}$-ray diffraction for phase analysis and to estimate the grain size along the direction normal to the film plane. All samples were found to have the cubic fluorite structure, as expected for the compositions produced. Samples produced with substrate temperatures $<550{ }^{\circ} \mathrm{C}$ were found to be nontextured, while a (100) texture was observed to develop at higher growth temperatures. Grain sizes were estimated using the Scherrer method. With increasing deposition temperature, the grain size increased smoothly and reproducibly from $10 \mathrm{~nm}$ for growth at $500{ }^{\circ} \mathrm{C}$ to about $100 \mathrm{~nm}$ at $600^{\circ} \mathrm{C}$, as seen in Fig. 1 .

Film cation composition was determined by $\mathrm{x}$-ray energy dispersive spectroscopy. If all parameters other than the substrate temperature were held constant, the yttrium content in the films was found to decrease with increasing growth temperature. To maintain an approximately constant compo- 


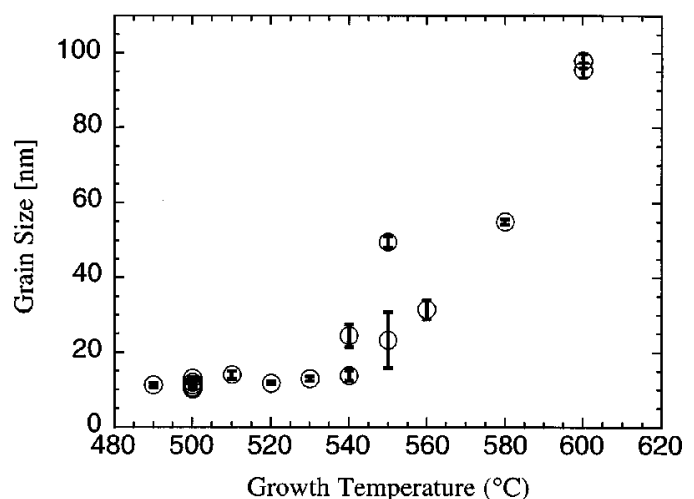

FIG. 1. Observed dependence of nanocrystalline YSZ grain size on substrate temperature during MOCVD growth.

sition for all films, the $\mathrm{Zr}$ bubbler pressure was systematically increased with increasing substrate temperature. This led to a $\mathrm{Y}_{2} \mathrm{O}_{3}$ content between 8 and $15 \mathrm{~mol} \%$ for all samples used in the thermal conductivity studies. Over this range of compositions, the change in thermal conductivity due to varying oxygen vacancy content is expected to be significantly smaller than the measured variations for different grain-sized samples. Changes in composition were also found to be uncorrelated with grain size, providing confidence that the observed changes were grain-size dependent rather than composition dependent.

The $3 \omega$ method, following Cahill and co-workers, ${ }^{8-10}$ was used to measure thermal conductivity. The method determines the thermal conductivity normal to the plane of the film by measuring the temperature rise of a thin metal line on the surface of the sample in response to an alternating current of frequency $\omega$. For the present study, $3 \omega$ data were obtained from samples over the temperature range $20-90{ }^{\circ} \mathrm{C}$, with drive frequencies of $10-1000 \mathrm{~Hz}$.

The thermal conductivity for both a $13 \mathrm{~nm}$ grain-sized YSZ film and an alumina substrate are shown in Fig. 2. The results indicate that over the narrow measured temperature range, the YSZ thermal conductivity is temperature independent, while the $\mathrm{Al}_{2} \mathrm{O}_{3}$ thermal conductivity decreases measurably with increasing temperature. The observed magnitude of this decrease in thermal conductivity of $\mathrm{Al}_{2} \mathrm{O}_{3}$ is in good agreement with expectations from literature values for $\alpha$-alumina. ${ }^{11}$

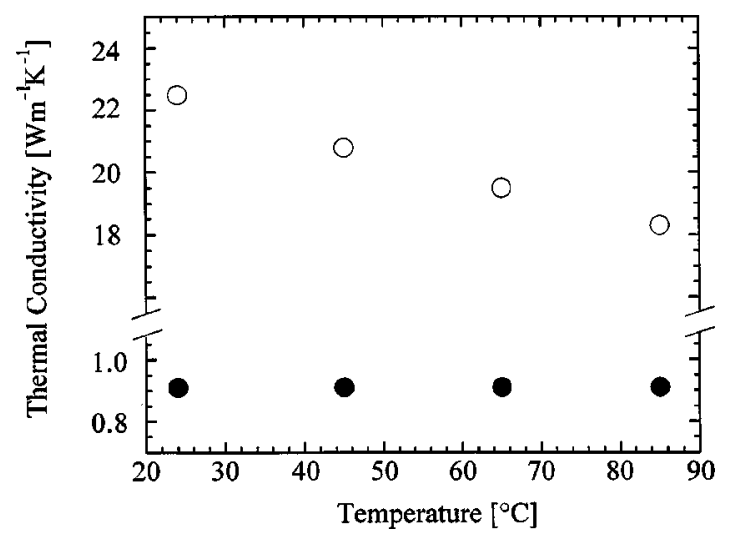

FIG. 2. Measured temperature dependence of the thermal conductivity of a $13 \mathrm{~nm}$ grain-sized YSZ film (closed circles) and the polycrystalline $\alpha-\mathrm{Al}_{2} \mathrm{O}_{3}$

optical refractive index values using the Lorentz-Lorenz
substrate (open circles).

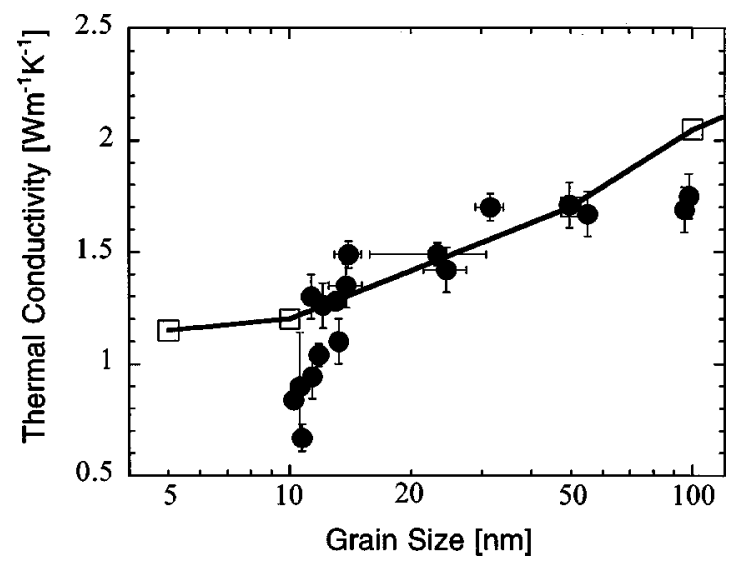

FIG. 3. Measured room temperature thermal conductivity values for YSZ thin films as a function of grain size (closed circles). The predicted behavior calculated by Klemens and Gell (Ref. 6) (open squares) for cubic YSZ containing 7 wt. \% yttria is shown for comparison.

The measured room temperature thermal conductivity values for YSZ thin films as a function of grain size are shown in Fig. 3. For all grain sizes larger than $30 \mathrm{~nm}$, the thermal conductivity is approximately constant and $\sim 20 \%$ smaller than the literature value for bulk YSZ. ${ }^{5}$ For smaller grain sizes, the thermal conductivity was observed to decrease rapidly with decreasing grain size, reaching a value of less than one-third the bulk value for the smallest grain-sized samples. While the measured thermal conductivity values are in good agreement with the predictions of Ref. 6 for grain sizes larger than $30 \mathrm{~nm}$, the observed rapid decrease in thermal conductivity for smaller grain sizes was not predicted. At the smallest grain sizes, the observed thermal conductivity values are approximately half the predicted values for the minimum conductivity amorphous limit of YSZ calculated using an Einstein model. ${ }^{9}$ This apparent discrepancy could be influenced by porosity in the samples as discussed below.

Only one previously published experimental report of thermal conductivity in nanocrystalline YSZ exists at this time. Raghavan et al. ${ }^{12}$ investigated the thermal conductivity of bulk YSZ samples prepared from sintered nanocrystalline powders with grain sizes as small as $30 \mathrm{~nm}$. The interpretation of the results in that study was complicated by the significant grain-size-dependent porosity found in all samples with grain sizes less than $70 \mathrm{~nm}$. In particular, they observed no effect of grain size on thermal conductivity for dense samples, but a significant dependence of the thermal conductivity on varying amounts of porosity for smaller-grained samples. The present results are in good agreement with Ref. 12 in that both studies found no effect of grain size on thermal conductivity for nanocrystalline samples with grain sizes larger than $\sim 30 \mathrm{~nm}$. The strong grain-size-dependent effect seen in the present study was observed only for samples with significantly smaller grain sizes than measured in Ref. 12 .

In light of the observations of Raghavan et al. ${ }^{12}$ that porosity provides a significant effect on thermal conductivity in nanocrystalline YSZ, it was important to investigate the potential role of porosity on the results of the current study. Two procedures were used to determine whether the density of the YSZ films varied with grain size during processing. In the first, the densities of films were calculated from measured optical refractive index values using the Lorentz-Lorenz 
TABLE I. Room temperature hardness and elastic modulus values for nanocrystalline YSZ obtained from nanoindentation studies.

\begin{tabular}{ccc}
\hline \hline Grain size $(\mathrm{nm})$ & Hardness $(\mathrm{GPa})$ & Modulus $(\mathrm{GPa})$ \\
\hline 12 & $5.8 \pm 0.5$ & $145 \pm 20$ \\
15 & 6.5 & 170 \\
20 & 6.7 & 150 \\
40 & 6.8 & 180 \\
100 & 5.7 & 130 \\
bulk (literature) & $12-17$ & $200-220$ \\
\hline \hline
\end{tabular}

law ${ }^{13}$ and the known refractive index dependence on composition for fully dense YSZ. ${ }^{14}$ Values of $93 \pm 2 \%$ of bulk density were found, with no measurable dependence of density on growth temperature.

Nanoindentation studies of mechanical behavior also provided insight into the possible presence of porosity in the YSZ films. As seen in Table I, it was observed that both the elastic modulus and the hardness of nanocrystalline YSZ coatings were smaller than values for bulk YSZ. Similar behavior has been observed previously for nanocrystalline ceramics ${ }^{15}$ and metals, ${ }^{16}$ and has been shown to correlate with the presence of porosity in samples. The observed reductions in Young's moduli are commensurate with expectations based on $\sim 10 \%$ porosity, ${ }^{17}$ especially if the pores are concentrated on the grain boundaries rather than randomly distributed. The Young's moduli were determined based on contact stiffness during unloading. In order to determine these values, it was necessary to account for the effects of the substrate. ${ }^{18,19}$ While reduced compared to bulk values, the measured modulus and hardness values did not vary significantly with grain size. The importance of this observation is that while the nanoindentation data appear consistent with the presence of some porosity in the YSZ films, the amount of porosity does not appear to vary significantly with grain size. Thus, it is concluded that the variations in thermal conductivity observed in the present study are more likely attributable to changes in grain size than changes in porosity.

A possible explanation for the observed grain size dependence of the thermal conductivity of YSZ can be provided by considering the relationship between the grain size of the samples and the phonon mean-free-path, $\lambda_{\mathrm{ph}}$, in single-crystal YSZ. Debye ${ }^{20}$ derived the relationship $\lambda_{\mathrm{ph}}$ $=\left(20 * T_{m} * d\right) /\left(\gamma^{2 *} T\right)$ to describe the temperature dependence of $\lambda_{\mathrm{ph}}$, where $T_{m}$ is the absolute melting temperature, $d$ is the lattice constant, and $\gamma$ is the Gruneisen constant. Using this relation, $\lambda_{\mathrm{ph}}$ for single crystal YSZ is calculated to equal $25 \mathrm{~nm}$ at $300 \mathrm{~K}$. It is reasonable to expect that, consistent with the present observations, reductions in thermal conductivity due to phonon scattering by grain boundaries will only become significant for grain sizes smaller than $\lambda_{\mathrm{ph}}$. Since $\lambda_{\mathrm{ph}}$ is temperature dependent, this explanation predicts that the critical grain size below which the thermal conductivity drops rapidly compared to the bulk conductivity will also depend on temperature. Measurements of the thermal conductivity of nanocrystalline YSZ over a much wider temperature range than that of the present study are in progress to test this hypothesis.

If the observed thermal conductivity behavior is due to a reduction in grain size below the phonon-mean-free path as proposed, a consequence in terms of the potential use of nanocrystalline YSZ for elevated temperature thermal barriers is that the required grain size for a particular reduction in thermal conductivity will decrease with increasing temperature. For example, $\lambda_{\mathrm{ph}}$ in $\mathrm{YSZ}$ at $1525^{\circ} \mathrm{C}$ is calculated to be only $4 \mathrm{~nm}$. The resistance of the nanocrystalline microstructure to grain growth at high temperatures will also be an important issue when considering potential TBC applications of nanocrystalline YSZ. The onset of rapid grain growth in the YSZ films of the present study was observed to occur at temperatures above approximately $900^{\circ} \mathrm{C}$.

In summary, it has been demonstrated that MOCVD processing can produce YSZ films with controlled composition and grain size. A significant reduction in thermal conductivity in these films compared to that of bulk YSZ is observed for grain sizes smaller than the phonon mean-free path. While there is evidence for porosity in the samples produced, the amount of porosity was not observed to vary significantly with grain size. Hence, the observed changes in thermal conductivity are more likely attributable to the effects of grain size than to porosity. These results demonstrate potential benefits of grain refinement to the nanometer scale in TBC applications, but also indicate that extremely small grain sizes will need to be produced and stabilized against grain growth in order to obtain significant thermal property improvements.

The authors thank David Cahill for his help and advice during the setup and testing of our $3 \omega$ system. Thanks also to Dean Miller for help with lithography and Dave Rinks for the use of the SEM. This work was supported by the U.S. Department of Energy, Office of Science, under Contract No. W-31-109-ENG-38.

${ }^{1}$ G. A. Kool, J. Therm. Spray Technol. 5, 31 (1996).

${ }^{2}$ W. P. Parks, E. E. Hoffman, W. Y. Lee, and I. G. Wright, J. Therm. Spray Technol. 6, 187 (1997).

${ }^{3}$ U. Betz and H. Hahn, Nanostruct. Mater. 12, 911 (1999).

${ }^{4}$ J. R. Weertman, D. Farkas, K. Hemker, H. Kung, M. Mayo, R. Mitra, and H. Van Swygenhoven, MRS Bull. 24, 44 (1999).

${ }^{5}$ D. P. H. Hasselman, L. F. Johnson, L. D. Bentsen, R. Syed, and H. L. Lee, Am. Ceram. Soc. Bull. 66, 799 (1987).

${ }^{6}$ P. G. Klemens and M. Gell, Mater. Sci. Eng., A 245, 143 (1998).

${ }^{7}$ Inorgtech, distributed by First Reaction, Hampton Falls, NH.

${ }^{8}$ D. G. Cahill, Rev. Sci. Instrum. 61, 802 (1990).

${ }^{9}$ D. G. Cahill, S. K. Watson, and R. O. Pohl, Phys. Rev. B 46, 6131 (1992).

${ }^{10}$ S.-M. Lee and D. G. Cahill, J. Appl. Phys. 81, 2590 (1997).

${ }^{11}$ M. Rohde and B. Schulz, J. Nucl. Mater. 173, 289 (1990).

${ }^{12}$ S. Raghavan, H. Wang, R. B. Dinwiddie, W. D. Porter, and M. J. Mayo, Scr. Mater. 39, 1119 (1998).

${ }^{13}$ M. Laube, F. Rauch, C. Ottermann, O. Anderson, and K. Bange, Nucl. Instrum. Methods Phys. Res. B 113, 288 (1996).

${ }^{14}$ D. L. Wood, K. Nassau, and T. Y. Kometani, Appl. Opt. 29, 2485 (1990).

${ }^{15}$ M. J. Mayo, R. W. Siegel, A. Narayanasamy, and W. D. Nix, J. Mater. Res. 5, 1073 (1990).

${ }^{16}$ G. E. Fougere, L. Riester, M. Ferber, J. R. Weertman, and R. W. Siegel, Mater. Sci. Eng. A 204, 1 (1995).

${ }^{17}$ W. D. Kingery, H. K. Bowen, and D. R. Uhlmann, Introduction to Ceramics, second edition (Wiley, New York, 1976), p. 776.

${ }^{18}$ D. S. Stone, J. Mater. Res. 13, 3207 (1998).

${ }^{19}$ M. F. Tambwe, D. S. Stone, J.-P. Hirvonen, I. Suni, and S.-P. Hannula, Scr. Mater. 37, 1421 (1997).

${ }^{20}$ G. H. Geiger and D. R. Poirier, Transport Phenomena in Metallurgy (Addison-Wesley, Reading, MA, 1973), p. 190. 